\title{
NOTAS SOBRE EL GENERO Geastrum PERS. EN CHILE CENTRAL
}

\section{(Notes on the genus Geastrum Pers. in Central Chile)}

\author{
Hugo Madrid L. \\ Unitat de Microbiologia, Facultat de Medicina i Ciències de la Salut, \\ Universitat Rovira i Virgili, Sant Llorenç 21, 43201 Reus (Tarragona), España. \\ Tel:34-977759350,E-mail: hugo.madrid@urv.cat
}

Palabras clave: Geastrales, Geastrum, Distribución Key words: Geastrales, Geastrum, Distribution

\section{RESUMEN}

Se reporta el hallazgo de Geastrum campestre, $\boldsymbol{G}$ fornicatum y $G$ pectinatum en la zona central de Chile. Junto a la descripción e ilustraciones de estas especies, se comentan aspectos de su ecología, taxonomía y distribución. G. campestre es nuevo para el catálogo micológico chileno.

\section{INTRODUCCION}

Geastrum Pers. (Geastrales) es un género cosmopolita de basidiomicetos gasteroides que crecen en suelo o madera, y que según Hawksworth et al. (1995) incluiría unas 50 especies. Las fructificaciones de este género son inicialmente globoides o con forma de bulbo, y su peridio consta de dos capas, denominadas exo- y endoperidio. En la madurez el exoperidio se rasga desde el ápice en forma estrellada, dejando ver el endoperidio, que libera los propágulos a través de un ostíolo apical rodeado de un peristoma (Calonge, 1996, 1998). Pese al llamativo aspecto de sus carpóforos, por el cual son conocidos en otros países como «estrellas de tierra» o «earthstars» (Gerhardt et al., 2000; Bates, 2004), y a ser hongos relativamente frecuentes en el centro y sur de Chile, las especies de Geastrum han sido aún poco estudiadas en el país (Montagne, 1850; Espinosa, 1917; Lazo, 1972, 2001).

El estudio taxonómico de basidiocarpos recolectados en Chile Central permitió identificar tres especies de Geastrum, Geastrum campestre Morgan, Geastrum fornicatum (Huds.) Hook y Geastrum pectinatum Pers. En el presente trabajo, junto a la descripción e ilustraciones de estas especies, se comentan aspectos sobre su ecología, taxonomía y distribución.

Recibido el 12 Mayo 2008

Aceptado el 23 Agosto 2008

\section{ABSTRACT}

Geastrum campestre, G. fornicatum, and G. pectinatum are reported from Central Chile. Descriptions and illustrations of these species are provided with comments on their ecology, taxonomy and distribution. $\boldsymbol{G}$ campestre is new to Chile.

\section{MATERIAL Y METODOS}

Los carpóforos fueron recolectados desde suelo, en las localidades de Santiago (Región Metropolitana) y Guacarhue (VI Región). Para la observación de estructuras microscópicas de las especies estudiadas, se realizaron preparaciones de la gleba en $\mathrm{KOH} \mathrm{3 \%} \mathrm{y} \mathrm{lactofenol.} \mathrm{Las}$ medidas de las esporas incluyen la ornamentación. Las colecciones se conservan en el herbario de la Sección Botánica del Museo Nacional de Historia Natural de Chile y en el herbario particular del autor.

\section{RESULTADOS Y DISCUSION}

Geastrum campestre Morgan, Amer. Naturalist 21: 1027. 1887 (Figuras 1 A-B)

Material estudiado: En suelo de un cerro, bajo Acacia caven (Mol.) Mol. Guacarhue, VI Región, Provincia de Cachapoal, Chile. 26-junio-2006. SGO153511.

Macroscopía: Carpóforos de 34-41 mm de diámetro x 14-16 mm de altura. Exoperidio rasgado en 7-9 rayos higroscópicos. La capa micelial adhiere abundantes restos vegetales y tierra. Capa carnosa de color café, persistente, agrietada. Capa fibrosa blanquecina. Endoperidio de color blanco sucio, globoide, sobre un corto pedicelo, áspero al tacto, de superficie verrugosa al examinarlo bajo la lupa. Peristoma fuertemente surcado. 
Microscopía: Esporas globosas de 6-8 $\mu \mathrm{m}$ de diámetro, verrugosas. Capilicio liso de 5-6 $\mu \mathrm{m}$ de diámetro, amarillento pálido.

Observaciones: G. campestre se diferencia fácilmente del resto de las especies de Geastrum citadas de Chile por su endoperidio fuertemente verrugoso (Calonge, 1998; Lazo, 2001). En el material estudiado, el endoperidio se mostraba muy áspero en estado fresco, cuando los basidiomas aún estaban bien hidratados por la humedad del suelo y por gotas de rocío. Sin embargo, tras secar los basidiomas esta cualidad se hacía menos evidente al tacto.

La literatura consultada menciona un hongo muy parecido a G. campestre, llamado G. pouzarii V.J. Staněk, conocido de Europa y Asia. Esta especie también posee rayos higroscópicos, peristoma surcado, endoperidio verrugoso y esporas fuertemente ornamentadas (Moreno \& Ladó, 1984; Calonge, 1996; Esqueda et al., 2003). Calonge (1998) señaló que, por su similitud con $\boldsymbol{G}$. campestre, $\boldsymbol{G}$. pouzarii debería considerarse como una variedad de dicha especie, para la cual propuso el nombre G. campestre var. pouzarii (V.J. Staněk) Calonge, caracterizada por sus esporas algo menores (5-7 $\mu \mathrm{m}$ diam.) y por la presencia de fisuras radiales en la cara externa de la capa fibrosa. Otras especies (aún no reportadas de Chile), con las cuales $\boldsymbol{G}$. campestre podría confundirse son $\boldsymbol{G}$. berkeleyi Massee y G. kotlabae V.J. Staněk. A nivel macroscópico, G. berkeleyi es muy similar a $\boldsymbol{G}$ c campestre, con endoperidio verrugoso y peristoma surcado, pero posee rayos no higroscópicos. Además sus esporas no superan los $7 \mu \mathrm{m}$ de diámetro (Calonge 1996; Bates, 2004). G. kotlabae tiene rayos higroscópicos y peristoma surcado, pero el endoperidio es farinoso y sus esporas tampoco exceden los $7 \mu \mathrm{m}$ de diámetro (Calonge, 1998).

G. campestre se ha reportado previamente de Estados Unidos, México, Australia, Europa, Sudáfrica y Bolivia (Bates, 2004; Rocabado et al., 2007). Esta especie crece en el suelo, en áreas despejadas, en pastizales o entre la hojarasca, bajo especies de Acacia Mill., Juniperus L., Pinus L., Prosopis L. y Quercus L. (Smith, 1951; Jeppson, 1987; Calonge et al., 1992; Moyersoen \& Demoulin, 1996; Calonge, 1998; Bates, 2004). El material estudiado, recolectado de suelo bajo Acacia caven («espino»), un árbol autóctono de la zona central de Chile, constituye un nuevo registro para el catálogo micológico nacional.

Geastrum fornicatum (Huds.) Hook., Fl. Londinensis 4: 575.1821 (Figura 1 C)

Material estudiado: En suelo, entre la hojarasca de planifolio no determinado. Santiago, Cerro San Cristóbal, Región Metropolitana, 4-octubre-2005. SGO153512.

Macroscopía: Carpóforos inicialmente globoidedeprimidos. En la madurez, los carpóforos abiertos alcanzan
39-54 mm de diámetro x 45-65 mm de altura. Exoperidio rasgado en 4 rayos no higroscópicos. La capa micelial forma en el suelo una estructura gruesa y resistente, similar a una copa, que adhiere restos vegetales, tierra y piedrecillas, sobre la cual se apoyan las puntas de los rayos de la capa fibrosa. Capa fibrosa de color café, con restos secos de la capa carnosa adheridos a su superficie. Endoperidio de 13-18 mm de diámetro x 9-12 mm de altura, globoide, de color gris a café oscuro, sobre un pedicelo de hasta $3 \mathrm{~mm}$ de longitud. Peristoma fimbriado, no delimitado.

Microscopía: Esporas de 4,3-5,8 $\mu \mathrm{m}$ de diámetro, globosas, verrugosas, de color café. Capilicio de 4-11 $\mu \mathrm{m}$ de diámetro, de color café, con paredes gruesas.

Observaciones: $\boldsymbol{G}$ fornicatum se caracteriza por poseer carpóforos abovedados, peristoma fimbriado, mal delimitado o sin delimitar, capa micelial ciatiforme hipogea y esporas verrugosas (Calonge, 1984; Ochoa, 1993; Bates, 2004; Leite et al., 2007). Es un hongo frecuente en el Cerro San Cristóbal, Santiago, donde fue observado en varias oportunidades entre los años 2005 y 2006.

En Chile se han reportado 2 especies de Geastrum con fructificaciones abovedadas, $\mathbf{G}$. fornicatum y $\mathbf{G}$. jurei Lazo, muy similares entre sí. De acuerdo a Lazo (1972, 2001), estos hongos pueden diferenciarse si se observan el endoperidio y el peristoma, siendo ambas estructuras concoloras en Geastrum fornicatum, mientras que en $\boldsymbol{G}$. jurei el peristoma es de color café grisáceo pálido y el endoperidio es café oscuro con tintes violáceos. Calonge (1998), en su monografía de hongos gasteroides ibéricos, menciona 2 especies adicionales, hasta el momento no reportadas de Chile, con las que podría confundirse $\boldsymbol{G}$. fornicatum, éstas son $\boldsymbol{G}$. welwitschii Mont. y $G$. quadrifidum Pers.:Pers. A diferencia de $\mathbf{G}$. fornicatum, $\mathbf{G}$ welwitschii posee una capa micelial ciatiforme epigea, que no adhiere restos del sustrato y tiene un rizomorfo bién desarrollado. Por otra parte, G. quadrifidum se distingue de $\boldsymbol{G}$. fornicatum por poseer un peristoma bien delimitado.

G. fornicatum es un taxón humícola que fructifica bajo planifolios, tales como Quercus, Acacia y Prosopis (Ochoa, 1993; Calonge, 1998; Bates, 2004). En Chile, este hongo se distribuye por las zonas central y austral (Lazo, 2001). G. fornicatum también se ha reportado de Estados Unidos, México, Europa, Sudáfrica, Australia, Brasil y Argentina (Ochoa, 1993; Calonge, 1998; Bates, 2004; Leite et al., 2007).

Geastrum pectinatum Pers. ex Pers., Syn. Meth. Fung.: 132.1801 (Figura 1 D-E)

Material estudiado: En suelo. Santiago, Cerro San Cristóbal, Región Metropolitana, 11-junio-2006. SGO 153513.

Macroscopía: Carpóforos de 28-38 mm de diámetro x 14-32 mm de altura. Exoperidio abierto en 10-12 rayos no 

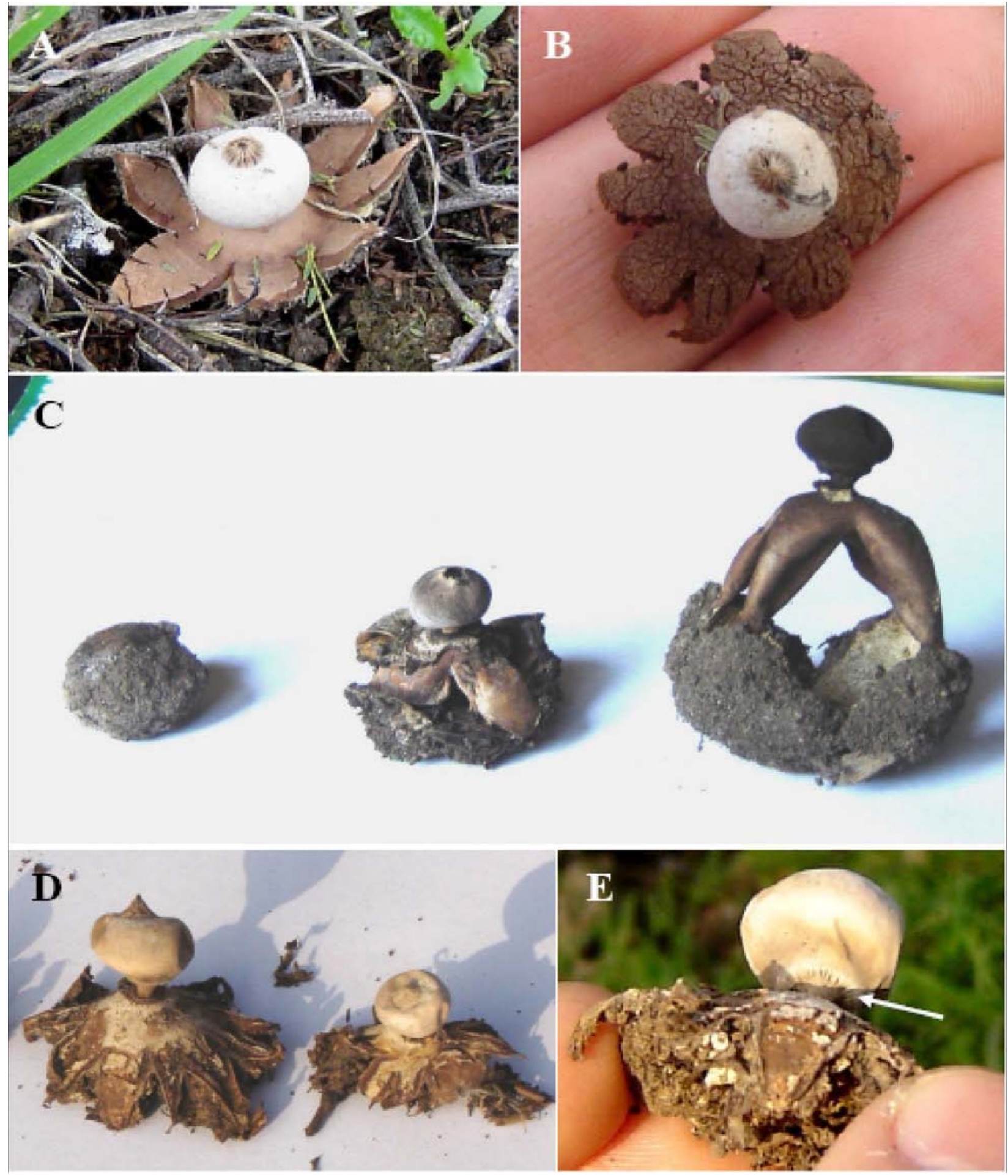

Figura 1. Basidiocarpos de: A, B. Geastrum campestre; C. G. fornicatum, en distintas etapas de maduración; D, E. G. pectinatum. La flecha en E señala las típicas estrías en la apófisis que caracterizan a esta especie.

higroscópicos, recurvados. La capa micelial adhiere abundantes restos vegetales y tierra. Capa fibrosa de color café, con la superficie interna parcialmente cubierta por restos secos de la capa carnosa. Endoperidio globoide, de color gris a beige, algo farinoso, de 10-13 $\mathrm{mm}$ de diámetro x 7-12 mm de altura, sobre un pedicelo de 1-2 mm de altura. Peristoma cónico, surcado. Apófisis estriada.

Microscopía: Esporas de 7- $8 \mu \mathrm{m}$ de diámetro, 
globosas, de color café, fuertemente verrugosas. Capilicio de 5-6 $\mu \mathrm{m}$ de diámetro, de color café claro, con paredes gruesas.

Observaciones: G. pectinatum es un hongo fácil de reconocer en el campo, caracterizado por sus rayos no higroscópicos, peristoma surcado y apófisis estriada (Breitenbach \& Kränzlin, 1986; Martín, 1988; Castro et al., 1993; Baseia et al., 2003; Calonge et al., 2005). La presencia de estrías radiales en la apófisis permite diferenciar a $\boldsymbol{G}$. pectinatum de especies similares, tales como $\mathbf{G}$. striatum DC. y G, nanum Pers. (no reportadas de Chile) (Calonge, 1998). Las dimensiones de las esporas muestran gran variabilidad en $\mathbf{G}$ pectinatum. A modo de ejemplo se puede mencionar que Baseia et al. (2003) encontraron, en material colectado en Brasil, medidas esporales de 4-4,5 $\mu \mathrm{m}$, incluyendo la ornamentación, mientras que Breitenbach \& Kränzlin (1986), en su monografía de hongos de Suiza, describen esporas de 4-6 $\mu \mathrm{m}$ de diámetro, sin considerar la ornamentación, compuesta por verrugas de hasta $1 \mu \mathrm{m}$ de longitud. El material chileno estudiado se acerca más a la descripción dada por estos últimos autores.

G. pectinatum crece en suelo. Según Baseia et al. (2003), la distribución de esta especie abarca Estados Unidos, México, Argentina, Brasil, Bélgica, Francia, España, Inglaterra, Noruega, Suecia, Islas Aland, Australia, Sudáfrica, El Congo y China. En Chile, Espinosa (1917) cita esta especie como Geaster pectinatus, sin precisar la localidad.

\section{AGRADECIMIENTOS}

A las señoras Elizabeth Barrera, Mélica Muñoz e Inés Meza, de la Sección Botánica del Museo Nacional de Historia Natural de Chile, por las facilidades otorgadas para el trabajo de laboratorio. Al Dr. Eduardo Piontelli, de la Facultad de Medicina de la Universidad de Valparaíso, por facilitar información sobre la distribución de especies estudiadas. A la señorita Laura Clavijo, por su colaboración técnica. A los expertos que han revisado el manuscrito original, por sus valiosos comentarios.

\section{REFERENCIAS}

Baseia, I.G.; Cavalcanti, M.A. \& Milanez, A.I. (2003) Additions to our knowledge of the genus Geastrum (Phallales: Geastraceae) in Brazil. Mycotaxon 85:409-416.

Bates, S. (2004). Arizona members of the Geastraceae and Lycoperdaceae (Basidiomycota, Fungi). Tesis para optar al grado de Master of Science. Arizona State University, Tempe.

Breitenbach, J. \& Kränzlin, F. (1986). Fungi of Switzerland. Vol. 2, Heterobasidiomycetes, Aphyllophorales and Gasteromycetes. Mycological Society of Lucerne. Lucerne.
Calonge, F.D. (1984). Adiciones y correcciones al catálogo del género Geastrum en España. Bol. Soc. Micol. Castellana 8:83-92

Calonge, F.D. (1996). Claves de identificación de los Gasteromycetes epigeos ibéricos. Bol. Soc. Micol. Madrid 21:359373

Calonge, F.D. (1998). Gasteromycetes I. Lycoperdales, Nidulariales, Phallales, Sclerodermatales, Tulostomatales. Flora Mycologica Iberica 3:1-271

Calonge, F.D.; Caballero, A. \& Palacios, J. (1992). Contribución al conocimiento de los hongos de La Rioja (Logroño, España). Gasteromycetes. Bol. Soc. Micol. Madrid 16:115-140

Calonge, F.D.; Mata, M. \& Carranza, J. (2005). Contribución al catálogo de los Gasteromycetes (Basidiomycotina, Fungi) de Costa Rica. An. Jard. Bot. Madrid 62:23-45

Castro, M.L.; Freire, L. \& Calonge, F.D. (1993). Catálogo provisional de los Gasteromycetes de Galicia (España). Bol. Soc. Micol. Madrid 18:87-104

Esqueda, M.; Herrera, T.; Perez-Silva, E. \& Sanchez, A. (2003). Distribution of Geastrum species from some priority regions for conservation of biodiversity of Sonora, Mexico. Mycotaxon 87:445-456

Espinosa, B.M. (1917). Informe del encargado de la sección plantas criptógamas. Bol. Mus. Nac. Chile 10:186-187

Gerhardt, E., Vila, J. \& Llimona, X. (2000). Hongos de España y de Europa. Manual de identificación. Ediciones Omega. Barcelona.

Hawksworth, D.L.; Kirk, P.M.; Sutton, B.C. \& Pegler, D.N. (1995). Ainsworth \& Bisby's dictionary of the fungi. 8 ed. CAB international, Wallingford.

Jeppson, M. (1987). Notes on some Spanish Gasteromycetes. Bol. Soc. Micol. Madrid 11:267-282

Lazo, W. (1972). Fungi from Chile I. Some Gasteromycetes and Agaricales. Mycologia 64:786-798

Lazo, W. (2001). Hongos de Chile. Atlas Micológico. Facultad de Ciencias de la Universidad de Chile. Santiago.

Leite, A.G.; Calonge, F.D. \& Baseia, I.G. (2007). Additional studies on Geastrum from northeastern Brazil. Mycotaxon $101: 103-112$

Martín, M.P. (1988). Aportación al conocimiento de las higroforáceas y los gasteromicetes de Cataluña. Vol. 2. Ediciones especiales de la Societat Catalana de Micología. Barcelona.

Montagne, C. En : Gay, C. (1850). Historia Física y Política de Chile. Botánica. Tomo 7. Museo de Historia Natural de Santiago; E. Thunot y Ca. París.

Moreno, G. \& Ladó, C. (1984). Estudios sobre el género Tulostoma y Geastrum (Gasteromycetes). Lazaroa 6:217-225

Moyersoen, B. \& Demoulin, V. (1996). Les Gasteromycetes de Corse : Taxonomie, ecologie et chorologie. Lejeunia n.s. 152:1128 
Ochoa, C. (1993). Contribución al estudio taxonómico, ecológico y corológico de la clase Gasteromycetes sensu lato en Baja California, México. Tesis de doctorado. Universidad de Alcalá de Henares, Alcalá de Henares.

Rocabado, D.; Wright, J.; Maillard, O. \& Muchenik, N. (2007). Catálogo de los Gasteromycetes (Fungi: Basidiomycotina) de Bolivia. Kempffiana 3:3-13
Smith, A.H. (1951). Puffballs and their allies in Michigan. University of Michigan Press, Ann Arbor. USA. 


\section{CURSO}

\section{HONGOS ENALIMENTOS Y AMBIENTES} INTERNOS

Universidad de Valparaíso, Escuela de Medicina, Cátedra de Micología. Valparaíso. Chile 3-7 Agosto 2009

Curso intensivo teórico práctico (41 horas), orientado a profesionales que se desempeñan en el area de la microbiología, para capacitar a profesionales (Ingenieros en alimentos, tecnólogos, biológos, químicos farmaceúticos u otros) en el manejo básico de la taxonomía fúngica a nivel de géneros y especies en hongos filamentosos.

\section{Contenidos del curso:}

Generalidades de los hongos, morfología, fisiología y ecología. Sistemas reproductivos, tipos de propágulos de dispersión, nociones de sistemática. Grupos taxonómicos a incluir: representates de los Mucorales, Eurotiales, Hypocreales, Sordariales y sus anamorfos asociados. Patrones de conidiogénesis, sistemática actual de los géneros Aspergillus, Penicillium y Fusarium. Métodos de cultivo.

Laboratorio: Manejo de lupa estereoscópica para la observación de colonias fúngicas en diferentes medios de cultivo y ambientes (aire, vegetación, alimentos, suelo, etc.). Preparaciones microscópicas para la identificación a nivel de género y especie en los medios específicos correspondientes. El laboratorio abarca el $80 \%$ de las horas del curso. Cupo: 20 personas.

Inscripciones e informaciones: Fono 32-2507370, Fax 322507375,@mail: anamaria.carreno@uv.cl 